\title{
EMPRESARIAS HISPANAS Y MEXICANAS EN ESTADOS UNIDOS Y MÉXICO'
}

\author{
ALICIA GIRÓN * \\ MIROSALBA LARA CANCINO **
}

\section{Resumen}

En las últimas dos décadas, la migración de mujeres empresarias presenta tasas de crecimiento superiores a las reportadas por los hombres. En este trabajo se observa cuáles son las características de las empresas encabezadas por mujeres hispanas, particularmente de México, durante los periodos comprendidos entre 1997-2002 y 2002-2007 de acuerdo con el último Censo de Estados Unidos; estos periodos corresponden al ciclo de crecimiento sostenido en Estados Unidos y México. Las notas comparativas entre estos dos periodos muestran una relación simétrica entre el número de mujeres empresarias migrantes, las oportunidades ofrecidas para los negocios y la tasa de crecimiento del producto interno bruto (PIB). La disminución de los negocios se presenta cuando aflora la crisis económica y financiera, tan profunda - o más - que la crisis de 1929. Justo a partir de este momento no sólo disminuyen los negocios de las mujeres migrantes empresarias, sino que también el largo fin de la crisis trastoca las esperanzas.

Palabras clave: migración femenina, mujeres empresarias, migrantes hispanas, Estados Unidos, México.

1. Este trabajo es resultado del Proyecto Conacyt: "Experiencia empresarial transfronteriza: repatriación de capitales para el desarrollo" [104503] realizado en el Instituto de Investigaciones Económicas de la Universidad Nacional Autónoma de México.

*Investigadora titular del Instituto de Investigaciones Económicas.

${ }^{\star *}$ Becaria del Proyecto Conacyt: "Experiencia empresarial transfronteriza: repatriación de capitales para el desarrollo”. 


\begin{abstract}
During the past two decades, entrepreneurial female migration has grown at a higher rate than that of men. This paper looks at the characteristics of businesses headed by Hispanic women, particularly from Mexico, during the 1997-2002 and 2002-2007 periods according to the last US Census; these periods belong within a cycle of sustained growth in both the United States and Mexico. Comparative notes between the two show a symmetric relation between the number of migrant businesswomen, business opportunities, and gross domestic product (GDP) growth rates. There has been a decline in business during the economic and financial crisis, which is as serious (perhaps even more so) than that of 1929 . This has led to a reduction in profits for migrant businesswomen, as well as prolonged crisis sequels that have reversed expectations.
\end{abstract}

Keywords: female migration, businesswomen, Hispanic migrants, United States, Mexico. 


\section{INTRODUCCIÓN}

l estudio sobre mujeres empresarias migrantes de origen hispano
en Estados Unidos desde de la perspectiva de la economía femi-
nista o del enfoque de género es un tema recientemente abordado a partir del crecimiento de pequeñas y medianas empresas encabezadas por empresarias. Los Objetivos de Desarrollo del Milenio de la Organización de las Naciones Unidas (ONU) contemplan a la mujer como una bandera necesaria para luchar contra la pobreza y se le concede una gran importancia como actriz dinámica en el desarrollo de las comunidades. $\mathrm{Al}$ respecto, hay dos elementos que es importante mencionar: primero, en el discurso oficial se refieren los presupuestos con enfoque de género y el acceso al crédito como mecanismos para el empoderamiento de las mujeres, y segundo, la mujer pasa a tener prioridad en la política fiscal, específicamente en el gasto público, y en la política financiera, mediante el otorgamiento de crédito y las microfinanzas. Con datos estadísticos de varios países:

se puede apreciar que entre el 25\% y el 33\% de las empresas del mundo están dirigidas por mujeres que son propietarias de las mismas. Estos negocios abarcan una gran diversidad de giros y tamaños - desde el nivel "micro" hasta las compañías multinacionales-y su presencia está transformando el panorama de la vida empresarial contemporánea (Zabludovsky y Grabinsky, 2001).

Según otros estudios, tan sólo en Estados Unidos 6.4 millones de mujeres son propietarias de sus empresas y dan trabajo a 9.2 millones de personas. En Europa, las mujeres empresarias dan empleo a 15 millones de personas, según estimaciones de la Organización para la Cooperación y el Desarrollo Económico (OCDE, 2004). Otros autores mencionan que las mujeres empresarias representan una parte importante dentro del conjunto de empresarios y han registrado un rápido crecimiento durante el periodo a estudiar. Algunos autores consideran que este desarrollo es una de las tendencias más notables en el mercado de trabajo de Estados Unidos (Efrat, 2010; Gibson, s.f.). 
Utilizando el Censo Económico de Estados Unidos -específicamente la Survey of Business Owners (SBO) - se observan datos estadísticos muy importantes: en la última década, las empresas encabezadas por mujeres hispanas y mexicanas han crecido a una tasa mayor que las de los hombres hispanos. Incluso los negocios emprendidos por estas mujeres han tenido tasas de crecimiento por arriba de la tasa de crecimiento de los negocios encabezados por mujeres en Estados Unidos.

Las empresas encabezadas por mujeres representaron el $28.7 \%$ de las empresas en Estados Unidos, de las cuales el 10.1\% correspondió a empresas de mujeres hispanas y mexicanas en 2007 (ver tabla 1). Es interesante observar que mientras la tasa media de crecimiento anual (TMCA) de las empresas en Estados Unidos fue de 2.0\% y 3.4\% durante los periodos 1997-2002 y 2002-2007, respectivamente, la de empresas propiedad de mujeres ha venido creciendo a un ritmo un poco mayor y de manera sostenida. En el periodo comprendido entre 1997-2007, la tasa de crecimiento representó el 3.7\% (ver tabla 2).

TABLA 1

Número de empresas en Estados Unidos 1997, 2002 y 2007 Absolutos

\begin{tabular}{|c|c|c|c|}
\hline & 1997 & 2002 & 2007 \\
\hline Total & $20,821,935$ & $22,974,655$ & $27,110,059$ \\
\hline Propiedad de mujeres & $5,417,034$ & $6,489,259$ & $7,793,384$ \\
\hline Propiedad de hispanas & 337,708 & 540,745 & 787,914 \\
\hline \multicolumn{4}{|c|}{$\begin{array}{l}\text { Participación porcentual } \\
\text { Respecto al total de empresas en E.U. }\end{array}$} \\
\hline & 1997 & 2002 & 2007 \\
\hline Total & 100.0 & 100.0 & 100.0 \\
\hline Propiedad de mujeres & 26.0 & 28.2 & 28.7 \\
\hline Propiedad de hispanas & 1.5 & 2.4 & 2.9 \\
\hline \multicolumn{4}{|c|}{ Respecto al total de empresas propiedad de mujeres } \\
\hline & 1997 & 2002 & 2007 \\
\hline Propiedad de mujeres & 100.0 & 100.0 & 100.0 \\
\hline Propiedad de hispanas & 6.2 & 8.3 & 10.1 \\
\hline
\end{tabular}

Fuente: Elaboración propia con datos de la SBO [http://factfinder.census.gov]. 
TABLA 2

Número de empresas en Estados Unidos. Total nacional y propiedad de mujeres 1997, 2002 y 2007 / TMCA 1997-2002, 2002-2007

\begin{tabular}{lcc}
\hline & TOTAL & $\begin{array}{c}\text { PROPIEDAD } \\
\text { DE MUEJERES }\end{array}$ \\
\cline { 3 - 3 } 1997 & 20821935 & 5417034 \\
2002 & 22821655 & 6489259 \\
2007 & 27110059 & 7793364 \\
\hline & & \\
TMCA 1997-2007 & 2.7 & 3.7 \\
TMCA 1997-2002 & 2.0 & 3.7 \\
TMCA 2002-2007 & 3.4 & \\
\hline
\end{tabular}

Fuente: Elaboración propia con datos de la SBO [http://factfinder.census.gov].

De acuerdo con Guzmán y Rodríguez:

En los últimos decenios, el número de empresas creadas o impulsadas por mujeres en todo el mundo ha experimentado un crecimiento importante. Ello ha favorecido la realización de diversos estudios en las características y resultados de las empresas, así como en las actitudes y estrategias empresariales adoptadas por los empresarios según su género (Guzmán y Rodríguez, 2008:381).

La participación de las mujeres dentro del total de empresarios en México también ha sido importante en los últimos años aunque, como se explicará más adelante, no es fácil hacer una comparación entre los empresarios y sus negocios en ambos países, a partir de la información estadística disponible de acuerdo al género.

\section{LOS EMPRESARIOS Y SUS NEGOCIOS EN ESTADOS UNIDOS}

Las TMCA del Producto Interno Bruto (PIB) real de Estados Unidos en los periodos de 2000-2002, 2002-2007 y 2007-2009 fueron de 1.5\%, $2.6 \%$ y $-1.4 \%$, respectivamente. Por supuesto, hay una relación asimétrica entre la TMCA del PIB y el crecimiento de los negocios de empresarios migrantes hispanos y mexicanos, que se demostrará a continuación. En esta parte se hará una comparación de las principales características 
de las empresas en Estados Unidos durante 1997, 2002 y 2007, según su distribución geográfica, sector de actividad y número de empleados, para posteriormente contrastarlas con los datos de las empresas propiedad de mujeres.

Durante 1997-2002, el número de empresas en Estados Unidos pasó de 20.8 millones a 22.9 millones, lo cual significa una TMCA de $2.0 \%$. Para 2002-2007 se puede observar un importante aumento al pasar de 22.9 millones a 27.1 millones con una TMCA de 3.4\% (ver tabla 2). Es importante señalar las semejanzas existentes entre los dos periodos: en el periodo 1997-2002 la distribución geográfica del 54\% de las empresas se concentró en los siguientes diez estados: California, Texas, Nueva York, Florida, Illinois, Pennsylvania, Ohio, Michigan, Nueva Jersey y Carolina del Norte; para el periodo 2002-2007, la distribución geográfica se comportó de forma similar, pues se concentró en los estados de California, Nueva York, Texas, Florida, Illinois, Pennsylvania, Ohio, Michigan y Georgia, los cuales reunieron un poco más del 55\% de empresas tanto en 2002 como en 2007. Es interesante observar que Nueva Jersey y Carolina del Norte constituyeron el estado número diez en importancia durante 2002, 1997 y 2007 respectivamente (ver tabla 3).

En lo que se refiere al empleo por estado durante los dos periodos que se están comparando, conviene hacer notar que siete de ellos continúan ocupando los primeros lugares, aunque con algunos cambios en su posición relativa: California, Nueva York, Florida, Illinois, Ohio, Michigan y Nueva Jersey. California sigue siendo el más importante y se mantuvo como el estado con un mayor número de trabajadores en las empresas de Estados Unidos. Esto lo demuestra la Encuesta a Dueños de Negocios durante los tres años que se están comparando (SBO, 1997, 2002 y 2007; ver tabla 4). Hay que destacar que los estados que concentran el mayor número de empresas en Estados Unidos también concentran el empleo, aunque con algunos cambios en su posición relativa. 
TABLA 3

Número total de empresas ${ }^{1}$ en Estados Unidos. Total nacional y estados seleccionados $(1997,2002,2007)$

\begin{tabular}{lccccccc}
\hline & \multicolumn{2}{c}{1997} & \multicolumn{2}{c}{2002} & \multicolumn{2}{c}{2007} \\
\cline { 2 - 7 } Estados Unidos & ABSOLUTO & PORCENTAJE & ABSOLUTO & PORCENTAJE $^{*}$ & ABSOLUTO & PORCENTAJE $^{*}$ \\
\cline { 2 - 7 } California & $\mathbf{2 0 , 8 2 1 , 9 3 4}$ & $\mathbf{1 0 0 . 0 0}$ & $\mathbf{2 2 , 9 7 4 , 6 5 5}$ & $\mathbf{1 0 0 . 0 0}$ & $\mathbf{2 7 , 1 1 0 , 3 6 2}$ & $\mathbf{1 0 0 . 0 0}$ \\
Florida & $2,565,734$ & 12.32 & $2,908,758$ & 12.66 & $3,426,952$ & 12.64 \\
Georgia & $1,301,920$ & 6.25 & $1,539,207$ & 6.70 & $2,010,405$ & 7.42 \\
Illinois & 568,552 & 2.73 & 674,521 & 2.94 & 901,625 & 3.33 \\
Michigan & 882,053 & 4.24 & 985,120 & 4.17 & $1,124,625$ & 4.15 \\
Nueva Jersey & 677,473 & 3.25 & 735,531 & 3.20 & 817,470 & 3.02 \\
Nueva York & 654,227 & 3.14 & 708,837 & 3.09 & 782,028 & 2.88 \\
Carolina del Norte & $1,509,829$ & 7.25 & $1,707,168$ & 7.43 & $1,957,597$ & 7.22 \\
Ohio & 570,484 & 2.74 & 642,597 & 2.80 & 799,346 & 2.95 \\
Pensilvania & 781,284 & 3.75 & $8,17,693$ & 3.56 & 898,664 & 3.31 \\
Texas & 837,756 & 4.02 & 874,255 & 3.81 & 982,288 & 3.62 \\
Suma & $1,525,972$ & 7.33 & $1,734,509$ & 7.55 & $2,166,128$ & 7.99 \\
Resto de estados & $\mathbf{1 1 , 3 0 6 , 7 3 2}$ & $\mathbf{5 4 . 3 0}$ & $\mathbf{1 2 , 6 5 8 , 5 9 9}$ & $\mathbf{5 5 . 1 0}$ & $\mathbf{1 5 , 0 8 5 , 1 6 7}$ & $\mathbf{5 5 . 6 4}$ \\
\hline
\end{tabular}

Notas:

*Porcentaje de casda estado con respecto al total de Estados Unidos.

1. Incluye empresas con y sin asalariados.

2. La suma se refiere a los diez estados que concentran el mayor número de empresas en cada año. Fuente: Elaboración propia con datos de la SBO [http://factfinder.census.gov].

TABLA 4

Número total de empleados ${ }^{1}$ en empresas ${ }^{2}$ en Estados Unidos, según estados seleccionados

\begin{tabular}{lclclc}
\hline & 1997 & & 2002 & & 2007 \\
\cline { 2 - 5 } Estads Unidos & $\mathbf{1 0 0 . 0}$ & Estads Unidos & $\mathbf{1 0 0 . 0}$ & Estads Unidos & $\mathbf{1 0 0 . 0}$ \\
California & 11.1 & California & 11.6 & California & 11.6 \\
Texas & 6.8 & Nueva York & 6.6 & Texas & 7.4 \\
Nueva York & 6.6 & Florida & 5.6 & Nueva York & 6.4 \\
Florida & 5.3 & Illinois & 4.6 & Florida & 6.1 \\
Illinois & 4.8 & Ohio & 4.2 & Illinois & 4.5 \\
Pensilvania & 4.8 & Michigan & 3.5 & Pensilvania & 4.3 \\
Ohio & 4.5 & Nueva Jersey & 3.2 & Ohio & 4.0 \\
Michigan & 3.7 & Georgia & 3.0 & Nueva Jersey & 3.1 \\
Nueva Jersey & 3.2 & Carolina del Norte & 2.9 & Michigan & 3.0 \\
Carolina del Norte & 3.0 & Massachusetts & 2.7 & Georgia & 3.0 \\
Suma & $\mathbf{5 3 . 6}$ & Suma & $\mathbf{4 7 . 9}$ & Suma & $\mathbf{5 3 . 5}$ \\
\hline
\end{tabular}

Notas:

1. Porcentaje de cada estado con respecto al total de Estados Unidos.

2. Incluye empresas con y sin asalariados.

Fuente: Elaboración propia con datos de la SBO [http://factfinder.census.gov]. 
Al comparar la distribución de las empresas en Estados Unidos según el sector de actividad, se presentan dos cuestiones interesantes; la primera es que durante 1997, el 63.5\% de estas empresas se concentró sólo en cinco sectores: "comercio al por menor", "servicios de apoyo a los negocios y manejo de desechos y servicios de remediación", "construcción", "otros servicios" y "servicios profesionales, científicos y técnicos". También se puede observar un comportamiento similar en la distribución por sectores de las empresas durante 2002 y 2007, así como una mayor distribución de los negocios entre las distintas actividades económicas, de manera que mientras cinco sectores de actividad concentraron el 63.5\% de los negocios en Estados Unidos en 1997, para 2002 los cinco sectores más importantes reunían el 58.6\%. En 2007, el 57.6\% de las empresas siguió concentrándose en cinco sectores. Por lo que puede inferirse, la tendencia a disminuir la concentración de empresas en pocos sectores continuará creciendo.

La otra cuestión a resaltar son los movimientos que han tenido los diferentes sectores. En primer lugar, "comercio al por menor", que concentraba el mayor número de empresas en 1997, ocupó el cuarto lugar en importancia para 2002 y 2007; "servicios de apoyo a los negocios y manejo de desechos y servicios de remediación" ocupaba el segundo lugar en 1997, y para 2002 y 2007 fue sustituido por “construcción", reduciendo su participación en más de $50 \%$. Por su parte, cabe destacar el incremento en la participación de "servicios profesionales, científicos y técnicos"; "construcción"; "servicios inmobiliarios y alquiler de inmuebles" y "otros servicios", que cobraron importancia después de 1997 (ver tabla 5).

Los sectores de actividad económica en los que se concentraron más empresas, tanto en 2002 como en 2007 son "servicios profesionales, científicos y técnicos"; "construcción"; "otros servicios excepto administración pública"; "comercio al por menor" y "servicios inmobiliarios y de alquiler de bienes muebles e intangibles". En relación con el ritmo de crecimiento durante 1997-2002, hubo cuatro sectores que crecieron de manera importante, entre $7.4 \%$ y $12.8 \%$ : "servicios profesionales,

1. Este renglón corresponde al subsector 56 dentro del Sistema de Clasificación Industrial de América del Norte [SCIAN] de México. 
científicos y técnicos", "servicios de esparcimiento, culturales y deportivos", "servicios financieros y de seguros" y "servicios inmobiliarios y de alquiler de bienes muebles e intangibles". Para 2002-2007, el ritmo de crecimiento de las empresas según el sector de actividad fue mucho menor que durante el periodo anterior; a pesar de esto, los sectores que registraron un mayor crecimiento en la cantidad de empresas fueron los de "dirección de corporativos y empresas y servicios de remediación", "transporte y almacenamiento" y "servicios de esparcimiento, culturales y deportivos", que crecieron a una TMCA de entre 5\% y 6\% (ver tabla 5).

Además, se presenta un cambio interesante en lo relativo a los sectores de actividad que absorbieron una mayor proporción de trabajadores durante el periodo. Siguen siendo cinco los sectores que reúnen el mayor número de empleados (cerca del 60\%). Sin embargo, todavía en 2002 la "manufactura" era el sector con el mayor porcentaje de éstos, mientras que los "servicios de salud y asistencia social" ocupaban el tercer lugar en importancia. Para 2007, ambos sectores intercambiaron posiciones (ver tabla 6). 


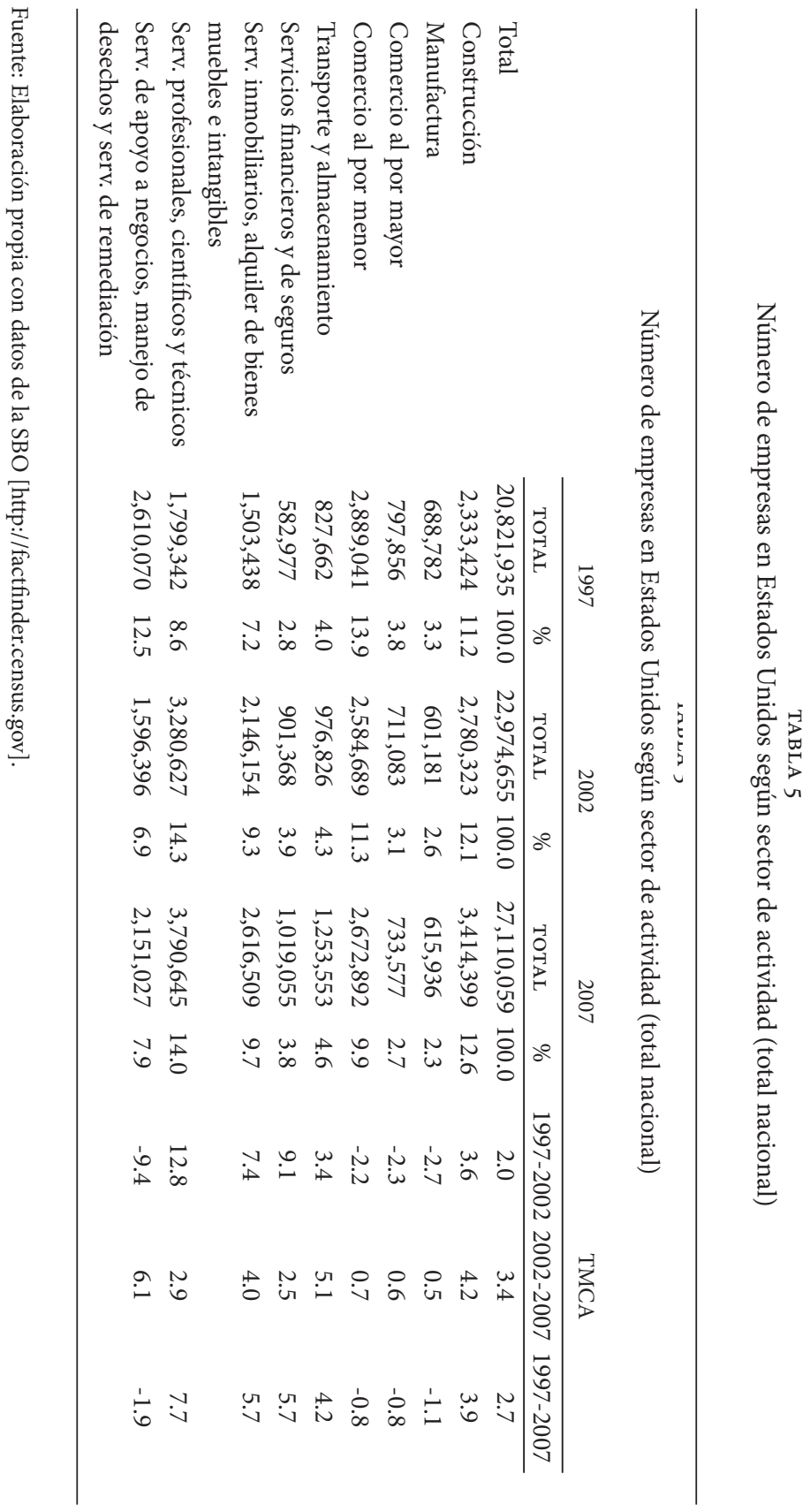


TABLA 6

Número de empleados en Estados Unidos por sectores de actividad ${ }^{1}$ seleccionados, 2002 y 2007

\begin{tabular}{|c|c|c|}
\hline \multirow[t]{2}{*}{ SECTORES } & \multicolumn{2}{|c|}{ NÚMERO DE EMPLEADOS } \\
\hline & ABSOLUTO & PORCENTAJE $^{*}$ \\
\hline Total & 110766605 & 100.00 \\
\hline Manufactura & 14992682 & 13.54 \\
\hline Comercio al por menor & 14757370 & 13.32 \\
\hline Cuidado de la salud y asistencia social & 13937400 & 12.58 \\
\hline Restaurantes y hoteles & 10253936 & 9.26 \\
\hline $\begin{array}{l}\text { Serv. de apoyo a negocios, manejo de } \\
\text { derechos y serv. de remediación }\end{array}$ & 8825729 & 7.97 \\
\hline Suma $^{2}$ & 62767117 & 56.67 \\
\hline \multicolumn{3}{|c|}{2007} \\
\hline \multirow[t]{2}{*}{ SECTORES } & \multicolumn{2}{|c|}{ NÚMERO DE EMPLEADOS } \\
\hline & ABSOLUTO & PORCENTAJE $^{*}$ \\
\hline Total & 118613426 & 100.00 \\
\hline Cuidado de la salud y asistencia social & 15908186 & 13.41 \\
\hline Comercio al por menor & 15630870 & 13.18 \\
\hline Manufactura & 13739543 & 11.58 \\
\hline Restaurantes y hoteles & 11654077 & 9.83 \\
\hline $\begin{array}{l}\text { Serv. de apoyo a negocios, manejo de } \\
\text { derechos y serv. de remediación }\end{array}$ & 10650672 & 8.98 \\
\hline Suma $^{2}$ & 67583348 & 56.98 \\
\hline
\end{tabular}

Notas:

*Porcentaje en cada estado con respecto al total de Estados Unidos.

1. North American Industry Classification System.

2. Se refiere a la suma de los cinco sectores que concentraron el mayor número de trabajadores.

Fuente: Elaboración propia con datos de la SBO [http://factfinder.census.gov].

\section{MUJERES EMPRESARIAS EN ESTADOS UNIDOS}

Después de observar brevemente las principales características de los empresarios y sus negocios durante 2002-2007, se hará una revisión para el caso específico de las mujeres, haciendo una comparación con el total nacional.

Según Lamolla (s.f.), hasta hace poco tiempo - escasamente dos décadas-, nadie se habría planteado la necesidad de abordar el estudio de las empresarias y sus diferencias con los empresarios, en parte debido a que, hasta entonces, las mujeres "eran prácticamente inexistentes" y 
permanecían en la invisibilidad. Hacia finales del periodo 2002-2007, el número de empresas propiedad de mujeres en Estados Unidos pasó de 6.4 millones a 7.7 millones, lo que representó una TMCA de 3.7\% que, como se ha mencionado, fue ligeramente mayor al crecimiento registrado por el total de las empresas en Estados Unidos.

Si se revisa por estados la concentración de las empresas propiedad de mujeres durante el periodo, puede observarse que siguen una tendencia similar a la del total de empresas en el país. Así, los estados que reúnen el mayor número de empresas de mujeres son California, Nueva York, Texas, Florida, Illinois, Ohio, Pennsylvania, Michigan, Georgia, Nueva Jersey y Carolina del Norte (ver tabla 7).

Los siguientes datos pueden dar una aproximación acerca de la importancia de los negocios propiedad de mujeres en relación con el total de empresas en Estados Unidos. Un primer dato resulta de relacionar el número de negocios propiedad de mujeres por estado respecto del total de empresas en ese mismo estado. Los resultados muestran que hay varios estados que, además de concentrar una proporción importante del total de empresas, están por arriba del promedio nacional: de $28.2 \%$ y $28.7 \%$ para 2002 y 2007, respectivamente. Para ambos años coinciden cuatro de los cinco principales estados: Washington, D. C., Maryland, Nuevo México y Hawaii. En quinto lugar se encontraron California (2002) y Georgia (2007) (ver tabla 7).

Un segundo dato resulta de observar cómo ha sido el crecimiento del número de empresas durante el periodo, para el total nacional y para las empresas propiedad de mujeres, ya que, aun cuando hay estados que no registran un número tan importante de negocios, sí han registrado un mayor ritmo de crecimiento. De acuerdo con esto, los tres estados que crecieron a una tasa más elevada coinciden para el total nacional y para el total de empresas de mujeres: Georgia, Nevada y Florida. Es interesante observar las cifras y notar que, en la mayor parte de los casos, la tasa de crecimiento de las empresas de mujeres resulta mayor. 


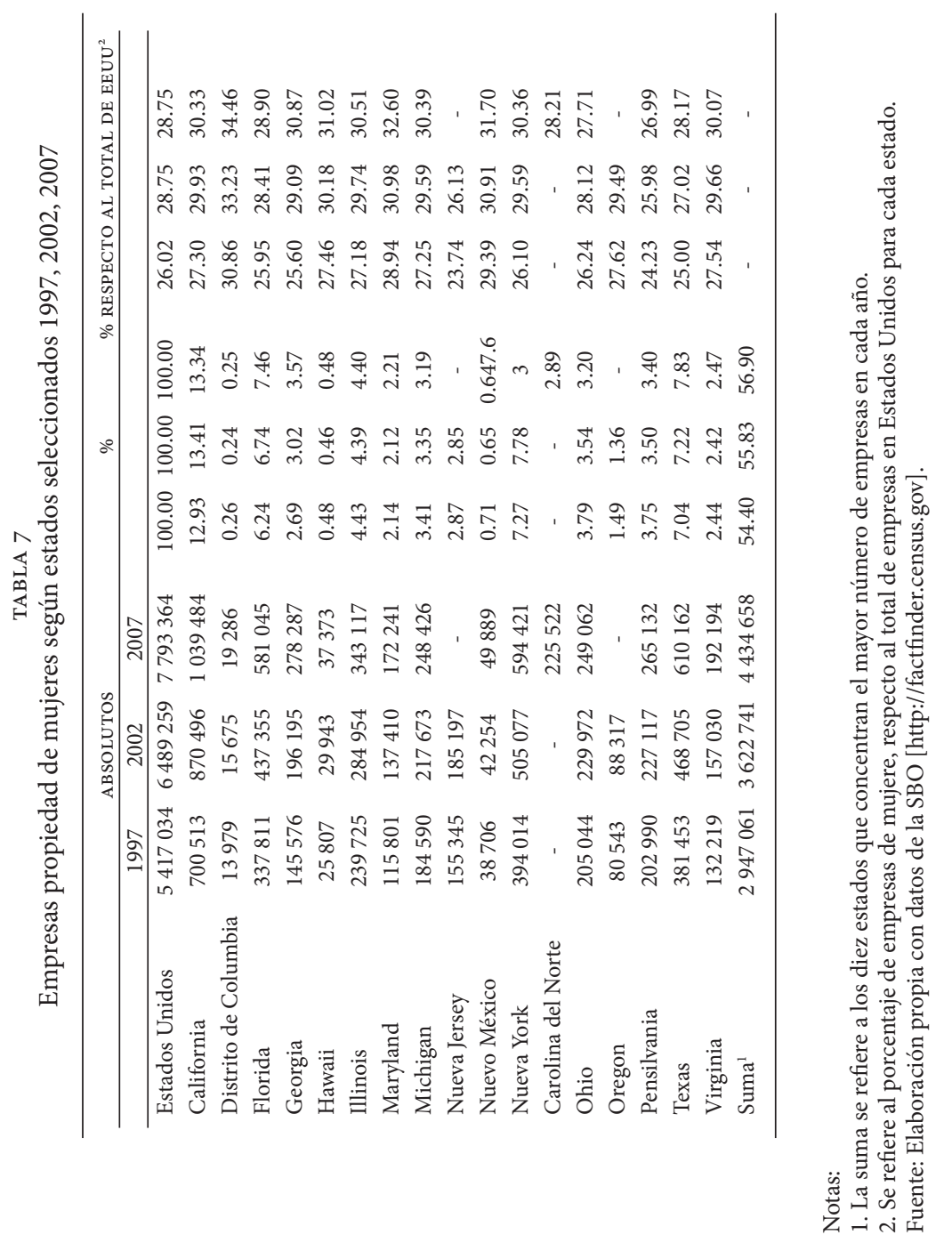


Las cinco actividades económicas en las que se reúne la mayor parte de empresas de mujeres en Estados Unidos durante el periodo en estudio son "servicios de salud y asistencia social", "otros servicios excepto administración pública", "comercio al por menor", "servicios profesionales, científicos y técnicos" y "servicios de apoyo a negocios y manejo de desechos y servicios de remediación".

\section{Semejanzas y diferencias: total nacional y empresas de mujeres}

Como se mencionó anteriormente, la localización geográfica de las empresas propiedad de mujeres sigue un comportamiento muy similar al del total de empresas en Estados Unidos; sin embargo, en la mayoría de los casos, las empresas propiedad de mujeres crecen a un ritmo mayor y concentran un porcentaje más elevado de negocios en comparación con el total nacional. Para Efrat, "this tremendous growth in female-owned businesses is due in part to various statutory enactments that have removed structural barriers women traditionally faced in owning a businesses" (Efrat, 2010:528). En tanto que:

much of the recent research explores the motivations, industry choices, and characteristics of women's entrepreneurs. (...) First, motivation is based on either necessity or opportunity. Although opportunity is generally the primary motivation, in middle-income countries, the ratio of opportunity to necessity entrepreneurship is much smaller for women than in high-income countries (Gibson, s.f.).

En relación con el tipo de actividades económicas desempeñadas por las empresas de mujeres en comparación con el total nacional, en ambos casos, "otros servicios", "comercio al por menor" y "servicios profesionales" se encuentran dentro de los cinco más importantes en términos del número de empresas; sin embargo, también hay una diferencia importante: mientras que dentro del total de empresas en Estados Unidos las otras dos actividades importantes son "construcción" y "servicios inmobiliarios", en el caso de las empresas propiedad de mujeres las otras dos actividades con mayor número de negocios son "apoyo a negocios" y 
"servicios de salud y asistencia social", siendo esta última la que ocupa el primer lugar en importancia - con 16\% del total de negocios de mujeres- y una actividad que ha sido considerada como "tradicionalmente femenina" (ver tabla 8).

En lo referente al número de trabajadores ocupados en las empresas propiedad de mujeres en 2002, se pudo observar la misma tendencia que en el caso del total de empresas en Estados Unidos: los sectores que absorbieron más trabajadores fueron los de "servicios de apoyo a negocios y manejo de desechos y servicios de remediación", "servicios de salud y asistencia social", "restaurantes y hoteles", "comercio al por menor" y "manufactura”. Sin embargo, una diferencia importante es que mientras para el total nacional los sectores con más trabajadores fueron "manufactura" y "comercio al por mayor", las empresas de mujeres tuvieron más empleados en los sectores de servicios en general ("apoyo a negocios", "salud y asistencia social" y "restaurantes y hoteles"), los cuales también presentan una mayor concentración de trabajadores en comparación con el total nacional (ver tabla 9).

\section{Características de las empresas de mujeres de origen hispano en Estados Unidos durante el periodo 2002-2007}

Durante 2002, se registraron 6.4 millones de empresas propiedad de mujeres en Estados Unidos, de las cuales 540.7 mil (8.3\%) pertenecían a hispanas -o latinas - y contaban con un total de 282.6 mil empleados, que corresponden al $4 \%$ del total del empleo en las empresas de mujeres en ese país. Esto podría indicar que, en términos generales, las empresas de mujeres hispanas o latinas son de menor tamaño en comparación con el resto de empresas de mujeres (ver tabla 10).

Las actividades económicas en las que se reúne el mayor número de empresas de hispanas son cinco: "servicios de salud y asistencia social", "otros servicios excepto administración pública", "servicios de apoyo a negocios y manejo de desechos y servicios de remediación", "comercio al por menor" y "servicios profesionales, científicos y técnicos", donde se concentra $77.2 \%$ de negocios de mujeres hispanas o latinas en ese país (ver tabla 10). 


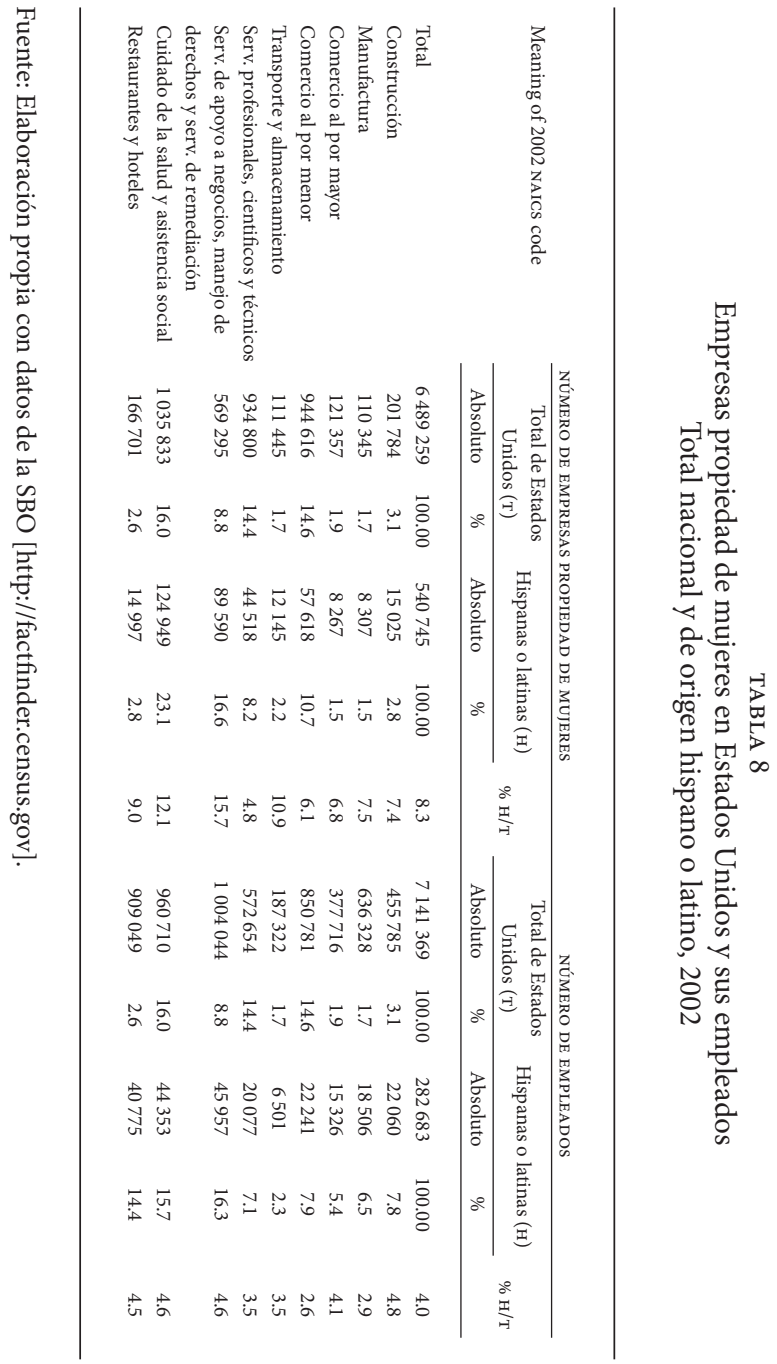




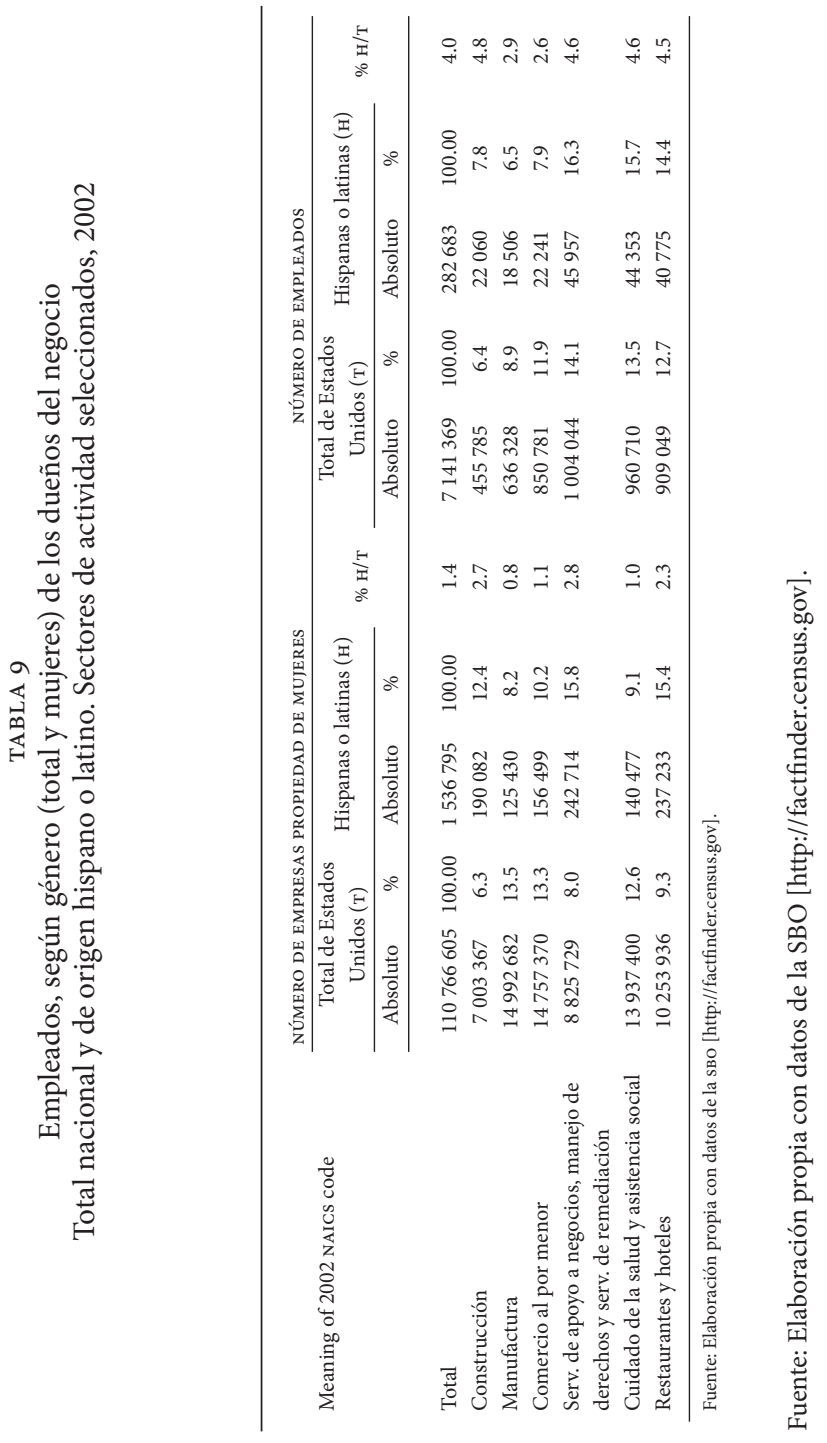


Si se comparan estos resultados con el resto de empresarias en Estados Unidos, puede observarse que también son éstas las actividades económicas en donde se encuentra el mayor número de empresas y que reúnen una proporción considerable del total de empresas, mayor a $50 \%$; no obstante, esta concentración es mucho más acentuada en el caso de empresas de mujeres hispanas o latinas que en el resto de empresas de mujeres: $69.3 \%$ para el total de empresas de mujeres, y $77.2 \%$ para el caso de empresas de dueñas hispanas o latinas.

Si se considera la importancia de las empresas de mujeres latinas en comparación con el total de empresarias en Estados Unidos, en términos del número de negocios según el sector de actividad económica, las cinco actividades más importantes en 2002 fueron las de "servicios de apoyo a negocios y manejo de desechos y servicios de remediación", "servicios de salud y asistencia social", "transporte y almacenamiento", "otros servicios excepto administración pública" y "restaurantes y hoteles", en las cuales se encuentra $57.6 \%$ de estos establecimientos (ver tabla 10).

Finalmente, en 2002 el empleo en los negocios de mujeres de origen hispano o latino presentó varias características interesantes: los cinco sectores que concentraron el mayor número de trabajadores fueron exactamente los mismos que para el caso de las empresas propiedad de hispanos, aunque con algunas diferencias en la posición relativa de cada uno. Mientras que en las empresas de latinos el empleo se concentró, por orden de importancia, en "servicios de remediación", "restaurantes y hoteles", "manufactura", seguidos de "comercio al por menor" y "servicios de salud y asistencia social", en las empresas de mujeres hispanas hay una concentración de trabajadores, considerablemente mayor, en los sectores de "servicios de remediación", "salud y asistencia social" y "restaurantes y hoteles", que nuevamente se encuentran dentro de las actividades consideradas como tradicionalmente desempeñadas por mujeres (ver tabla 9). 


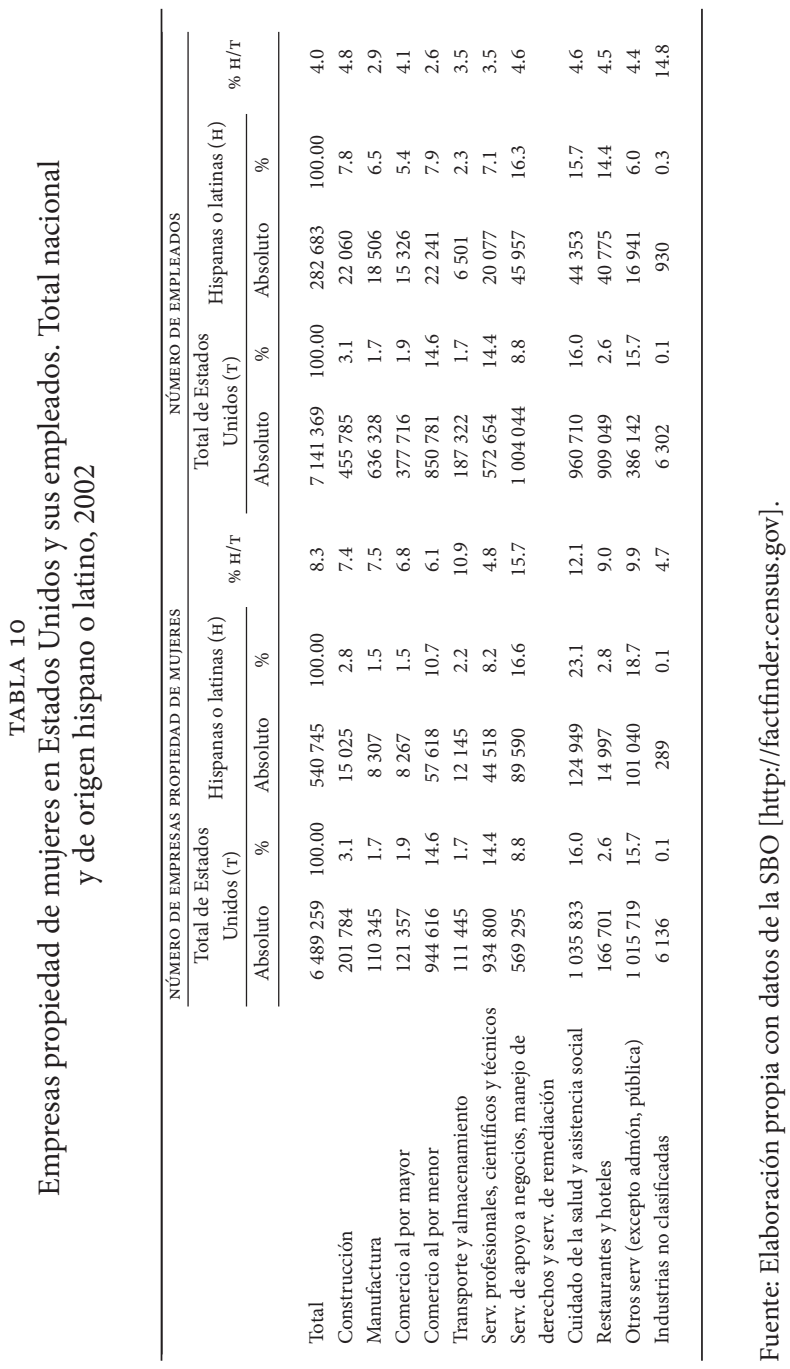


Debido a que la información específica sobre el origen étnico (detallado) según género del dueño del negocio sólo aparece desagregada a partir de 2007, en esta parte se presentará únicamente un panorama acerca de las empresarias mexicanas en Estados Unidos durante este año:

- Dos estados concentran cerca del 70\% de las empresas de mexicanos: California y Texas;

- El número total de empresas propiedad de mexicanos y el de empresarias mexicanas sigue el mismo comportamiento y se concentra en dos estados: California y Texas;

- En el caso de los empresarios mexicanos y las empresas de propiedad mixta (hombre-mujer), California y Texas siguen siendo los estados que concentran el mayor número de empresas; en orden de importancia, Texas es el primero, seguido por California, y

- Hay cuatro estados en los que más de la mitad de las empresas de mexicanos son propiedad de mujeres: Hawaii, Massachusetts, Distrito de Columbia y Connecticut.

\section{EMPRESARIAS EN MÉXICO}

Como se mencionó anteriormente, no es fácil hacer una comparación entre los datos de empresarios y sus negocios en México y en Estados Unidos, debido a que la información no es del todo disponible; sin embargo, se tratará de presentar algunos datos que nos permitan hacer una comparación aproximada. ${ }^{2}$ De acuerdo con la Encuesta Nacional

2. Como ya se ha dicho, se utilizó como fuente de información estadística la Encuesta a Dueños de Negocios (Survey of Business Owners [SBO]) para el caso de los dueños de negocios en Estados Unidos. En el caso de México, no se cuenta con una encuesta de este tipo; existen varias encuestas por tipo de actividad económica (comercio, manufactura, transportes, servicios, etcétera), pero la información no viene desagregada por género del propietario. En este sentido, la información que se consideró como más cercana a lo que se deseaba comparar fue la proporcionada por las encuestas de empleo (antes Encuesta Nacional de Empleo [ENE], recientemente Encuesta Nacional de Ocupación y Empleo [ENOE]), donde es posible analizar datos de la población ocupada según "posición en el trabajo" y género. Sin embargo, es importante tener presente que 
de Empleo de 2002 elaborada por el Instituto Nacional de Estadística Geografía e Informática (INEGI), del total de empresarios, 15\% eran mujeres. Si se compara este dato con el hecho de que, para ese mismo año, del total de empresas en Estados Unidos, el 28.2\% era propiedad de hombres, puede observarse que las mujeres empresarias tienen una mayor importancia relativa en ese país. Aunque si se compara cómo han crecido los negocios de mujeres en Estados Unidos en relación con el total y cómo ha aumentado la cantidad de empresarias en México en relación con el total, se observa un significativo crecimiento en el número de empresarias en México.

De acuerdo con las cifras, durante el periodo comprendido entre 1997 y 2002, el total de empresarios en México presentó una TMCA de $0.31 \%$, mientras que las empresarias crecieron a una tasa del 5.39\%. Para 2002-2007, las tasas fueron del $4.59 \%$ y $9.34 \%$, respectivamente. De ahí que la importancia de las empresarias en México durante estos periodos no radica en su volumen, sino en el rápido crecimiento que han registrado en estos años, por lo que se concluye que las mujeres empresarias mexicanas, al igual que las mujeres hispanas en Estados Unidos, están accediendo a encabezar negocios como respuesta a las oportunidades para instalar pequeñas y medianas empresas en un ciclo ascendente de crecimiento.

\section{LA CRISIS Y ALGUNAS CONCLUSIONES}

Indudablemente, la crisis financiera que aflora en 2007 en el sector de las hipotecas afecta no sólo a las mujeres empresarias de origen hispano en mayor medida que a los negocios encabezados por hombres, sino que trastoca las ganancias, el empleo y parte de los ingresos que envían a sus familiares en su país de origen. Más allá de la crisis y el cierre de negocios, las mujeres se volvieron empresarias porque había la necesidad de incrementar los ingresos familiares que desde hacía años venían mermando los ingresos de las familias. Es importante mencionar que

la información presentada no es estrictamente comparable, pues se trata de datos diferentes (número de empresas y número de empresarios). 
muchas de ellas cuentan, cada vez más, con un grado de escolaridad mucho mayor que el de los hombres, lo cual brinda la posibilidad de una mejor administración en los negocios. Las redes sociales que se establecen a partir de las escuelas, la iglesia y la propia familia han permitido que las mujeres logren mejores relaciones, no sólo a través de los clubes de migrantes (representados en su generalidad por hombres).

A falta de datos del impacto de la crisis, sólo nos resta señalar la importancia del crecimiento de las empresas de mujeres en un periodo de tasas de crecimiento sostenidas de la economía de Estados Unidos. De acuerdo con la SBO de 2002, el total de empresas en Estados Unidos fue de 22.9 millones, de las cuales 6.4 millones (28.2\%) eran propiedad de mujeres; de éstas, 540.7 mil (8.3\%) pertenecían a mujeres hispanas. Para 2007, la información preliminar de esta encuesta indica que el total de empresas fue de 27.1 millones; de éstas, 7.7 millones pertenecían a mujeres hispanas. Es interesante observar que mientras la TMCA de las empresas en Estados Unidos fue de 3.4\%, la de empresas propiedad de mujeres lo hizo a un ritmo un poco mayor: $3.7 \%$.

Al comparar la participación de empresarias "hispanas" en relación con el resto de mujeres dueñas de negocios en Estados Unidos, se observa que los sectores de actividad en los que se concentran estas empresas -que incluso sobrepasan el promedio nacional (8.3\%) - son "servicios de apoyo a los negocios y manejo de desechos y servicios de remediación" (15.7\%), "servicios de salud y asistencia social" (12.1\%), "transportes, correos y almacenamiento" (10.9\%), "restaurantes y hoteles" y "otros servicios" (con cerca del 10\% cada uno). Al mismo tiempo, es interesante observar que si se compara el número de empleados en estas empresas en relación con el total de empresas propiedad de mujeres en el país, la participación es mucho menor (cerca del 4\%), lo que podría indicar que se trata, en general, de pequeñas empresas.

Las empresas de mujeres hispanas por sector de actividad se concentran en los sectores de "otros servicios", "servicios de salud y asistencia social" y "servicios de apoyo a los negocios y manejo de desechos y servicios de remediación", pero aquí destaca también la participación de empresas en el sector "comercio" y "servicios profesionales, científicos y técnicos". Es interesante observar que, en términos generales, las 
empresas propiedad de mujeres siguen la misma tendencia en términos de localización geográfica que el resto de las empresas en Estados Unidos, pero tienden a concentrarse aún más que en el caso del total nacional y a tener ritmos de crecimiento mayores.

Las empresas de mujeres de origen hispano -o latino- presentan, en general, características y comportamientos semejantes a los de las empresas de hispanos (localización geográfica, actividades económicas preponderantes, etcétera) pero, al mismo tiempo, comparten otras características comunes a las del total de mujeres empresarias: mayor ritmo de crecimiento, mayor concentración de empresas o empleados en las actividades económicas preponderantes, mayor concentración en actividades "tradicionalmente desempeñadas por mujeres", entre otras.

\section{REFERENCIAS}

BArros Nock, Magdalena (2006), "Entrepreneurship and gender relations: The case of Mexican migrants in rural California”. Women's Entrepreneurship: Issues and Policies. Segundo Coloquio Internacional sobre Migración y Desarrollo: Migración, Transnacionalismo y Transformación Social.

Efrat, Rafael (2010), "Women entrepreneurs in bankruptcy" en Tulsa Law Review (45.3), pp. 527-550; disponible en http://www.csun.edu/ re38791/ pdfs/Women\%20Entrepreneurs\%20in\%20Bankruptcy\%20Final.pdf.

Gibson, Amy (s.f.), "Cultural Value Differences and Women-Owned Businesses in the United States: A Preliminary Exploration" en Journal of Applied Business and Economics, disponible en http://www.na-businesspress.com/ gibsonweb.pdf.

Girón, Alicia y Ma. Luisa González Marín (2004), "Mexican Women Migration: An Employment Right Lost", disponible en http://www.aliciagiron.com/ category/migracion.

Lamolla, Laura (2007), "Emprendedoras y emprendedores: ¿Cuestión de género?" en Boletín CIES (46), Centro de Investigación de Economía y Sociedad, CIES; disponible en http://www.grupcies.com/boletín/es/boletines-2009/112-boletines-2007.html.

OECD (2004), Conference of Ministers Responsible for Entrepreneurship and Innovative Small and Medium-sized Enterprises [SMEs]: Promoting in a Global Economy: towards a more responsible and inclusive Globalisation, Istanbul. 
U.S. CENSUS BUREAU (2006), Hispanic-Owned Firms: 1997, 2002 y 2007. Economic Census, SBO [Company Statistics Series], USA, Economics and Statistics Administration, Department of Commerce, 633 pp.; disponible en http://www.census.gov/econ/sbo/>.

Zabludovsky Kuper, Gina y Salo Grabinsky (2001): Mujeres, empresas y familias. Las Mujeres empresarias en México: una fuerza económica emergente, México, UNAM/ Del Verbo Emprender. 